\title{
L'ABBE JEAN-BAPTISTE DUBOS ET LA PRESSE
}

L'Abbé Jean-Baptiste Dubos ${ }^{1}$ naquit à Beauvais en 1670 et mourut à Paris en 1742. Fils d'un commerçant il vint faire ses études à Paris. Après avoir été reçu bachelier de Sorbonne en 1691 il entra dans le bureau des affaires étrangères. Il fut chargé d'affaires importantes dans differentes cours d'Europe, en Allemagne, en Italie, en Angleterre, et en Hollande. Il s'en acquitta fort heureusement, et fut nommé pour ses services, Abbé de Rezons, près de Beauvais. Il se consacra à la littérature et à l'histoire et entra en 1720 à l'Académie française.

Ses ouvrages ${ }^{2}$ sont une preuve de la variété et de l'étendue de ses connaissances. Ses Réflexions critiques sur la poésie et la peinture furent très appréciées dès leur publication. Le biographe Feller (384-85) réserve un paragraphe à ce sujet:

C'est un des livres les plus utiles en ce genre qu'on ait jamais écrits sur ces matières chez aucune des nations de l'Europe. Ce qui fait la beauté de cet ouvrage, dit l'auteur du Siècle de Louis XIV, c'est qu'il n'y a que peu d'erreurs et beaucoup de reflexions vraies, nouvelles et profondes. Il manque cependant d'ordre et surtout de précision; mais l'écrivain pense et fait penser. Il ne savait pourtant pas la musique, il n'avait jamais pu faire des vers, il n'avait

${ }^{1}$ François Xavier de Feller. Biographie universelle ou dictionnaire historique, nouvelle édition augmentée par M. Pégennes, Tome IV, (Besançon: Outhenin Chalandre fils, 1842): 384-385.

${ }^{2}$ Histoire des quatre Gordiens, 1695. Vindiciae pro quatuor Gordianorum historia, 1700. Les interêts de l'Angleterre mal entendus dans la guerre présente, 1704 (il s'agit de la guerre de la Succession d'Espagne). Manifeste de Maximilien, électeur de Bavière contre Léopold, empereur d'Autriche, 1705. Histoire de la Ligue de Cambrai, 1709. Réflexions critiques sur la poésie et la peinture, 1719, 2 vol.; 1770, 3 vol. Histoire critique de l'établissement de la monarchie française dans les Gaules, 1734,3 vol. in $-4^{\circ}$. 
pas un tableau; mais il avait beaucoup lu, vu, entendu, et refléchi. La littérature ancienne lui était aussi connue que la moderne et les langues savantes et étrangères autant que la sienne propre.

D’Alembert à laissé sur Dubos le jugement suivant ${ }^{3}$ :

L'Abbé Dubos est un de ces hommes de lettres qui ont eu plus de mérite que de réputation ... S'il eut le mérite de joindre la philosophie au savoir, d'autres ont été ou des savants plus profonds ou des philosophes distingués ... Dans les beaux arts, il s'est montré un excellent juge, d'autres en ont expose les principes avec moins de sagacité peut-être mais avec plus d'éloquence ou d'agrément.

Ses contemporains reconnurent d'emblée la valeur des Réflexions critiques. Comme nous le verrons les journaux ne seront pas sans contribuer à sa notoriété, spécialement le Journal des sçavans de 1719 et de 1727 et le Journal litteraire de 1720.

Pour l'Abbé Dubos l'art et par là le beau, a une fonction libératrice. Il part du principe que le grand ennemi de l'homme est l'ennui. Pour le combattre il nous faut des passions or elles ont des suites dangereuses. C'est alors qu'intervient l'art: le beau produit des raisons "artificielles" capables de nous occuper dans le moment où nous les sentons et "incapables" de nous causer dans la suite des peines réelles. L'art imite les passions dont notre âme ne peut se passer tout en leur enlevant le caractère nocif: il épure les passions tout en les rendant avec intensité. Les conséquences de ce principe sont bouleversantes: la hiérarchie des genres est renversée, l'élégie est supérieure au drame car elle nous touche davantage et finalement le pouvoir de la peinture est plus grand que celui de la poésie car la peinture agit sur nous par le sens de la vue sans employer les signes artificiels auxquels la poésie est obligée de recourir; elle opère directement. Un élément non plus "sentimental" mais "sensuel" entre ainsi dans la nature du beau. Nous assistons à un glissement des valeurs rationnelles aux valeurs émotives et Dubos va jusqu'à soupçonnerl'existence d'un sixième sens, réserve à l'appréhension du beau.

${ }^{3}$ Georges Grente. Dictionnaire des lettres françaises, le VXIII ème siècle, Tome l. (Paris: Fayard, 1960): 396-97. 
Le Journal des sçavans des mois d'octobre et de novembre 1719 donne ce que le journaliste appelle un "extrait"4 qui ressemble à une revue telle que nous la trouvons dans les journaux littéraires avec quelques citations du texte original. L'auteur de l'article mentionne le manque d'organisation du texte des Réflexions mais cela ne semble nullement le gêner, au contraire voici ce qu'il en dit:

$\ldots$ il (Dubos) use de la liberté dont on connaissait si bien les
avantages dans les siècles plus florissants de la Grece et de Rome (JS
$19,498-509)$.

La popularité des Réflexions et la notoriété de Dubos viennent du fait qu'il ne se pose pas en savant mais en simple "amateur." Il admet que le public se compose d'individus tous aussi différents les uns que autres et il se propose de remonter dans son enquête jusqu'à la source du plaisir que nous procurent les oeuvres d'art.

L'auteur propose ainsi son dessein: J'ose entreprendre d'expliquer l'origine du plaisir que nous font les vers et les tableaux. Des entreprises moins hardies, passent pour être téméraires puisque c'est vouloir rendre compte a chacun de son approbation et de ses dégoats; c'est vouloir instruire les autres de la manière dont leurs propres sentiments naissent en eux. Ainsi je ne saurais esperer d'être approuvé, si je ne parviens point à faire connaître au lecteur dans mon livre ce qui se passe en lui-même en un mot, les mouvements les plus intimes de son coeur. On n'hésite guère à rejeter comme un miroir infidèle, le miroir où l'on ne se reconnaft pas (JS 19, 465).

C'est en parlant du "Génie" que le journaliste aborde le problème du beau.

L'étude perfectionne le Génie qu'elle ne donne point. Le Génie est une plante qui pousse d'elle-même mais dont la qualité des fruits dépend de la culture. Cette culture au reste n'a rien de gênant: le Beau se fait d'abord sentir à ceux qui sont nés pour l'exprimer (JS 19, 502).

C'est tout ce que dit l'article mais il s'agit d'une réflexion qui fait

${ }^{4} J o u r n a l$ des Sçavans 1719, Tome 66, (Edition d'Amsterdam: chez les Janssons): 446. 
penser. Puisque le journaliste l'utilise aussi librement c'est que l'idée du Beau était un concept familier au public des lecteurs. Cela ne veut pas dire que ce même public était capable de circonscrire ce qu'il entendait par le Beau. Ce qui est étonnant par contre c'est que le journaliste souligne que ce Beau "se fait sentir par son contraire" (JS 19, 503); cela est curieux car "le Laid" deviendra en effet un thème largement exploité par Baudelaire par exemple un siècle plus tard.

Dans un autre parágraphe le journaliste rapporte le fait que le génie est limité: chacun à sa spécialité. De plus le génie peut se permettre d'être "plagiaires" car, indique le journaliste:

Les vrais génies se saissisent des differentes beautés qu'ils y découvrent sans que pour cela ils encourent aucun blâme (JS 19, 505).

Mais ajoute-t-il, Dubos considere que tous les pays ne sont pas susceptibles de produire des génies.

En examinant l'effet des causes physiques, on observe qu'il est des pays où les Arts ne sauraient pour ainsi dire ni naître, ni fleurir (JS 19, 506).

Il en est ainsi des pays du Nord: la Hollande, l'Angleterre par exemple. Ainsi, bien avant la lettre, Dubos pose le problème du "lieu" et du "moment"; il ne néglige pas la "race," non plus, puisque le journaliste mentionne que selon Dubos, notre constitution physique influe sur notre production intellectuelle (JS 19, 506).

Le Journal des sçavans donne une idée générale du contenu des Réflexions de Dubos mais sans insister sur l'élément qui rendit cet auteur célèbre dans la postérité, à savoir l'existence en nous d'un sixième sens qui nous fait percevoir ce qui est Beau. Le journaliste se perd dans des détails intéressants certes, mais sans portée réelle. Il faudra attendre 1726 pour que le même Journal decouvre ce point essentiel du livre.

En 1727 le Journal des sçavans fait publier un article sur la Dissertation de Bel sur les pensées de Dubos. Bel était un conseiller au Parlement de Bordcaux d'où il était natif et membre de l'Académie de 
cette ville. Né en 1693, il mourut à Paris à l'âge de quarante-cinq ans d'un excès de travail. Il avait, selon Feller (Tome IV, 186) une très belle bibliothèque qu'il voulait rendre publique avec des fonds pour l'entretien des bibliothécaires. En 1726 il publia une dissertation sur les Réflexions où il examinait "le système de M. l'Abbé Dubos touchant la préférence que l'on doit donner au goat sur la discussion, pour en juger des ouvrages de l'esprit."

Selon le journaliste, Bel, dans sa "dissertation" questionne le sentiment de Dubos lorsque ce dernier prétend qu'il est plus sûr de juger des ouvrages par goût et par sentiment que par examen et par discussion en sorte que le jugement du public est préférable à celui des gens de métier que Dubos appelle des artisans. Le journaliste explique ce que Dubos entend par le mot "public." Par ce terme, Dubos entend les personnes du monde qui ont de l'éducation, de la littérature, du discernement, d'où s'est formé en eux un certain goût de comparaison. Le journaliste cite Dubos lui même à l'appui de son affirmation:

Il est en nous un sens destiné pour juger du mérite de ces ouvrages, qui consistent en l'imitation des objets touchants de la nature. Ce sens est le sens même qui aurait jugé de l'objet que le peintre ou le poète, le musicien ont imité. C'est l'oeil, lorsqu'il s'agit d'un tableau ... lorsqu'il s'agit de connaître si l'imitation qu'on nous présente dans le poème est capable d'attendrir le sens destiné pour en juger est le sens même qui aurait été attendri: c'est le sens qui aurait jugé de l'objet imité. C'est le sixieme sens qui est en nous, sans que nous voyons ses organes. C'est la portion de nous-même qui juge sur l'impression qu'elle ressent (JS 27, 157).

Le journaliste rapporte le sentiment de Bel sur ce sujet: pour Bel l'oeil ne juge pas réellement d'un tableau. C'est l'âme qui à l'occasion de l'objet et de la sensation forme son jugement. Le journaliste expose le raisonnement de Bel et le cite mot pour mot:

Qu'est-ce donc ce sixième sens qui nous est inconnu, selon lui et dont nous ne voyons pas les organes? N'est-ce point transporter aux belles lettres les qualités occultes de la vieille philosophie? Si ce sixième sens ne juge que sur l'impression qu'il reçoit ce jugement sera

\footnotetext{
${ }^{5}$ Continuation des mémoires de litterature et d'histoire. Tome II, part I (paris: Simart).
} 
souvent faux car un grand nombre de causes étrangères contribueront à cette impression, elle dêpendra aussi de la disposition intérieure, du degré de sensibilité de nos passions (JS 27, 157).

\section{Selon le journaliste Dubos affirme que:}

Le mérite le plus important des poèmes est de nous plaire; c'est le but principal que les poètes se proposent. On connaît donc suffisa mment s'ils ont bien réussi quand on connañ si l'ouvrage touche (JS 27, 158).

Le même journaliste cite l'opinion de Bel en ces termes:

Quoi qu'un ouvrage ait plu, son mérite est encore suspect. Les poésies de Ronsard et celle de M... ont eu l'avantage de plaire pendant quelques temps; la réputation de Ronsard a même survécu à ce poète. Cependant les poésies de ces deux auteurs ont maintenant peu de partisans; donc la fortune présente d'un ouvrage ne doit pas nous précipiter brusquement à le croire bon (JS 27, 159).

Après avoir fait état des deux opinions le journaliste se livre assez humoristiquement à une réflexion personnelle:

Pourquoi certaines personnes qui ne connaissent ni principes ni règles jugent-elles presque toujours sainement des ouvrages d'esprit et pourquoi en même temps d'autres personnes qui ont beaucoup d'esprit et de lumières et qui savent parfaitement les principes et les règles de chaque genre jugent-elles souvent mal? Il me semble que $\mathrm{M}$. Bel aurait da s'objecter cette difficulté et la ré-soudre. En attendant qu'il le fasse ... ne peut-on pas dire que ces personnes qui n'ont ni principes ni régles et pourtant jugent bien ont en elles-mêmes des principes et des règles de pratique et d'habitude? Elles jugent par comparaison, par imitation, par réminiscence car il faut supposer qu'elles ont du monde et de la lecture: leur goat, leur sentiment renferme donc une espèce de discussion virtuelle (JS 27, 159).

Le journaliste admet l'existence de personnes qui malgré leur connaissance des principes et des règles jugent mal. Dans ce cas il faut dire qu'elles ne perçoivent point ou que leur perception est confuse. La bonne discussion supposera toujours la perception. Le journaliste termine son article avec beaucoup d'esprit sur l'observation suivante:(JS 27,159 ) 
Le goat sans la discussion et la discussion sans le goat (c'est à dire le sentiment) sont des guides également infideles. Un bon juge est celui qui sent comme une femme d'esprit et raisonne comme un homme éclairé. (Nous avons pris la liberté d'ajouter ces observations à l'esprit de M. Bel).

Le journaliste, tout en restant courtois, est du côté du public et de Dubos et se moque un peu de l'Académicien de Bordeaux qui veut tout faire passer au crible de la raison seule. Pour Bel, ce que Dubos appelle "sentiment" n'est qu'une "discussion prompte." Mustoxidi dans son Histoire de l'esthétique française de 1700 a 1920, (30) mentionne un autre passage significatif de Bel à cet effet:

Ce sentiment n'est que l'impression produite, dans leur esprit par une comparaison subite et des idées qu'elles reçoivent de l'ouvrage, et de celles qu'elles ont acquises, soit par la réflexion, soit par la lecture, soit par le commerce du monde. Or c'est là une discussion marquée.

Pour Bel le sentiment n'est qu'une "discussion prompte" ou encore un "jugement superficiel" et il conclut en disant: (31)

Une discussion exacte et approfondie est supérieure à une discussion subite et momentanée.

Mustoxidi est de l'avis que Bel n'arrive qu'à montrer son incompréhension absolue du systeme de Dubos. Cela est possible mais il se peut également qu'il s'agisse d'un problème de terminologie. Les deux hommes parlent de la même chose avec des termes différents. Ce "sixième sens," dont Dubos fait mention n'est qu'une métaphore utilisée pour donner plus de force à sa pensée mais étant donné la qualité du "public" dont il est question, il est évident comme le souligne le journaliste d'ailleurs, que l'on peut aussi donner à ce "sixieme sens" l'attribut de discussion "virtuelle" ou "prompte" ou "instantanée." Bel, académicien de province, est le porte parole de l'esprit académicien traditionneldu XVIII ème siècle commençant. Dubos représente l'esprit amateur du XVIIIème siècle à Paris.

Le Journal littéraire de 1720 consacre une trentaine de pages (212-41) au compte rendu du premier volume des Réflexions de Dubos. 
Cet article nous apprend que l'ouvrage fut publié sous l'anonymat, en effet voici ce qu'il dit en terminant:

Nonobstant la modestie qu'a eue l'auteur de ne pas mettre son nom à un ouvrage si judicieusement écrit, on n'a pas eu de peine a connaftre qu'il était de M. l'abbe Dubos, à qui le public est redevable de l'Histoire de la Ligue de Cambrai, et de quelques autres ouvrages. Il ny à guère que lui qui puisse parler si savamment du genie et du goot des Anglais et le comparer avec celui des autres Nations, pour éclaircir et approfondir comme il fait, une si belle et si riche matière.(241)

Le but des Réflexions selon le Journal littéraire est le même que pour le journaliste du Journal des sçavans: il s'agit pour Dubos de tâcher d'éclaircir et d'expliquer l'origine du plaisir que font les vers et les tableaux (JL 20,213). Le journaliste résume la pensée de Dubos en disant d'abord que les vers et les tableaux plaisent en affligeant et il ajoute aussitôt que ces vers et ces tableaux servent à exciter des passions utiles. Voici une partie de son commentaire:(JL 20, 213)

Selon lui (Dubos), le mérite des tableaux et des vers consiste principalement à imiter les objets qui auraient excité en nous des passions réelles. Ces imitations ont beaucoup de pourvoir sur nous et le coeur humain en est facilement ému. Platon ne bannit les poètes de sa République que parce qu'il trouvait que leurs imitations peuvent faire trop d'impression.

Le journaliste explique comment Dubos répond à ce préjugé de Platon: (JL 20, 214)

Ces impressions excitęnt en nous des passions louables, telles que sont l'amour de la patrie et de la gloire, l'habitude de ces passions nous rend capables de bien des efforts de vertu et de courage que la raison seule ne pourrait pas nous faire tenter.

Après avoir ainsi développé l'utilité de l'excitation des passions, le journaliste nous entretient des sujets propres à la poésie et à la peinture. Dubos insiste, nous dit-il, sur des sujets "intéressants" qui puissent émouvoir et attacher; certains sujets sont plus avantageux pour les poètes que pour les peintres: le "qu'il mourût" du viel Horace par exemple. Par contre certains sujets sont plus avantageux pour les peintres: souvent le 
peintre choisit des objets que le poète tragique n'ose montrer (JL 20, 241). Le journaliste relève ensuite les pensées de Dubos relatives au sentiment de l'amour dans les tragédies; il distingue entre l'amour et la galanterie, jargon plein de fadeur qui rend les héros modernes peu propres à la tragédie. Quant à la comédie, Dubos considère qu'elle doit être prise des moeurs mêmes du peuple qui la voit (JL 20, 225).

Le journaliste suit fidèlement les Réflexions dans leurs méandres. Selon le journal, Dubos commente très justement la célèbre expression des partisans des Anciens: "tout est dit." A ce sujet Dubos affirme que "les peintres et les poètes qui ont précédé les nôtres n'ont pas épuisé les sujets." Enfin le journaliste consacre toute une longue section de son article à ce que Dubos appelle "la poésie de style": élément qui fait la destinée des poèmes. Le journaliste explique la pensée de Dubos comme suit: (JL 20, 232)

Nous admirons plusieurs poèmes qui ne sont rien moins que réguliers, mais qui sont soutenus par l'invention, et par un style plein de poésie, qui de moment en moment présente des images qui nous rendent attentifs et qui nous émeuvent.

Parmi les exemples que Dubos utilise pour démontrer la véracité de ce qu'il affirme, le journaliste choisit deux tragédies de Corneille:

Nous avons deux tragedies du Grand Corneille dont la conduite et la plupart des caractères sont très défectueux, à savoir $L e$ Cid et La Mort de Pompée. Mais la poésie du style en est telle que le public, malgré leurs défauts ne se lasse point de les admirer, et il les place fort au-dessus de plusieurs autres dont les moeurs sont meilleures et le plan plus régulier.(JL 20, 233)

Le journaliste réitère l'importance de "la poésie de style" sur quatre pages de son article et il conclut en ces termes:

Si donc l'on veut rappeler les choses à leur véritable principe. C'est par la poésie de style qu'il faut juger d'un poème plutôt que par sa régularité et par la décence de ses moeurs.(JL 20, 234)

La poésie étant constamment mise en parallèle avec la peinture, il est naturel que le journaliste nous entretienne de cette dernière. La 
poésie du style a son équivalent en peinture: il s'agit de "l'expression"; "la mécanique" de la poésie trouve le sien dans "le coloris."

L'expression paraît à notre auteur être dans la peinture ce que la poésie du style est dans le poème; et il compare le coloris avec cette partie de l'art poétique, qui du choix et de l'arrangement des mots forme des vers harmonieux. C'est ce qu'il appelle la mécanique de l'art.

L'article se termine sur des détails relatifs à la peinture et la sculpture ancienne et à la déclamation. Le compte rendu du second Tome est annoncé pour une date ultérieure. Ce compte rendu paraîtra douze années plus tard avec un bref résume du premier volume (JL 32, 171-185). Enfin le journalist s'évertue à dire "qu'il n'y a rien de plus raisonnable" que les règles de Dubos.

L'article nous semble superficiel car là où l'on s'attend à des commentaires, nous ne trouvons qu'une table des matières faite d'une succession de titres. Mais le journaliste fait des citations qui peuvent sembler insignifiantes pour nous mais qui sont représentatives de l'intérêt de cette epoque. C'est ainsi qu'il cite un paragraphe ayant trait aux organes: l'oeil, l'ouïe et leur développement entre l'âge de quinze à trente ans.

Les organes contractent sans peine durant ces années toutes les habitudes dont leur première conformation les rend susceptibles. (JL 32, 178).

Le mot "sentiment" donne lieu à une autre citation d'importance: peut être la plus célèbre de toutes les Réflextions de Dubos.

Non seulement le public juge d'un ouvrage sans intérêt, mais il en juge encore ainsi qu'il en faut décider en général, c'est à dire, par la voie du sentiment et suivant l'impression que le poème ou le tableau font sur lui. Puisque le premier but de la poésie et de la peinture est de nous toucher, les poèmes et les tableaux ne sont de bons ouvrages, qu'à proportions qu'ils nous émeuvent et qu'ils nous attachent. Or, le sentiment enseigne bien mieux si l'ouvrage touche

'Ibid., page 235. 
et s'il fait sur nous l'impression que doit faire un ouvrage que toutes les dissertations composées par les critiques pour en expliquer le mérite et pour en calculer les perfections et les défauts. La voie de discussion et d'analyse, dont se servent ces messieurs est bonne, à la verite, lorsqu'il s'agit de trouver les causes qui font qu'un ouvrage plaît ou qu'il ne plaît pas; mais cette voie ne vaut pas celle du sentiment, lorsqu'il s'agit de décider cette question. L'ouvrage plaît-il ou ne plaîtil pas? L'ouvrage est-il bon, ou mauvais, en genéral? C'est la même chose ... La décision de cette question n'est point du ressort du raisonnement. Il doit se soumettre au jugement que le sentiment prononce. C'est le juge compétent de la question. Raisonne-t-on pour savoir si un ragoat est bon ou s'il est mauvais? (JL 32, 182-83). impersonnel.

Pour le reste le jugement du journaliste est bref, elogieux mais

Le reste de cet ouvrage, et en général tout ce que nous n'avons fait qu'indiquer n'est pas moins digne de la curiosité du public, que ce qu'on vient de lire; et nous nous faisons d'autant plus de plaisir de le reconnaître ici, qu'on avait oublié d'en parler dans ce journal, lors de la première édition (JL 32, 184).

Bien que l'article soit fait d'une compilation de citations sans commentaire, il offre un certain intérêt de par la sélection des passages. Le langage de la critique confondait souvent "émotion," "sentiment" et "passion." Or les passions étaient mal vues et le devoir de chaque individu était de les réfréner. De par le choix des passages de Dubos on voit que la sensibilité a définitivement pris un caractère positif (JL 32, 184).

Le succès de Dubos est dû au fait qu'historiquement il survient à un moment où la critique cartésienne ne tient plus. A. Lombard dans son ouvrage: l'Abbé Dubos, un initiateur de la pensée moderne (183), explique ce phénomène en ces termes:

La critique cartésienne s'était d'abord conciliée avec le culte des Anciens parce que les Anciens étaient la source de la vérité et de la raison. Mais elle ne s'en était pas tenue là. Leur appliquant le critère des règles qu'ils avaient eux-mêmes fournies, elle avait constaté qu'ils nous étaient sur plusieurs points inférieurs. Les partisans des Modernes avaient ainsi ... introduit dans la critique l'idée de progrès. Mais leur jugement ne s'était affranchi de l'autorité des grands modèles que pour s'asservir plus étroitement à l'autorité abstraite des règles et de la raison. Ils ont prolonge si loin les applications de la 
méthode cartésienne qu'elles ont enfin révolté l'instinct litteraire et le bon sens. Et ainsi les défenseurs de la raison ont précipité la réaction du sentiment.

Que faut-il entendre par sentiment? Depuis longtemps les critiques s'étaient aperçu que le plaisir de l'art n'est pas facile à définir et qu'il comporte des élements dont l'analyse rationnelle ne rend pas compte et que la connaissance des règles ne fait pas le poète. L'Académie avait reconnu dans Le Cid un "agrément inexplicable." Cette part d'inconnu qui échappe aux règles avait été surnommé le "je ne sais quoi" et nous place dans le domaine du sentiment on plutôt du "goût." Lombard explique la situation au XVII ème sieccle:

Les classiques auraient admis volontiers que le godt n'est autre chose que l'intelligence éclairée par les règles en les appliquant instinctivement ou inversement, les règles c'est le goat explique, amené aux principes du beau et du vrai mais à des principes universels et permanents. Voila pourquoi Fontenelle ne veut pas qu'on méprise les règles sous prétexte qu'ily a un art de plaire; cet art lui aussi à ses règles (Lombard, 183).

Le goût était toujours considéré comme une résultante des facultés de l'esprit. Quclques amateurs de peinture, comme Roger de Piles font prévaloir le goût individuel. Mais la littérature était moins libre. Fénélon tout en reconnaissant que les Anciens étaient plus simples, plus grands, plus près de la nature que les modernes, affirme que la véritable puissance de la poésic repose sur l'émotion. Dubos, lui, pense à un "sixieme sens," sens distinct de la raison et juge de l'oeuvre d'art. La sensibilité selon Dubos qu'est-elle donc? C'est la vie même de l'âme aux prises avec les passions. Dubos maintient fermement que l'esprit ne peut connaître les passions que le coeur n'a pas ressenties.

Après le long article de 1720 , le Journal littéraire consacre quatorze pages de son Tome XIX, (Partie I) de 1732 aux Réflexions critique sur la poésic et las peinture. Cet article est publié à l'occasion d'une nouvelle édition revue et corrigée faite à Utrecht la même année. Ce qui nous frappe tout d'abord en comparant les deux articles à douze ans d'intervalle, c'est la longucur des citations prises directement chez Dubos. Le second article est pratiquement composé de citations. D'autre part le premier article s'attardait sur le premier volume qui traite 
de la relation entre la poésie et la peinture, du plaisir né de l'affliction, de l'excitement des passions, des sujets propres à ces deux arts, de l'intérêt des sujets choisis, de sújets avantageux pour les poètes et d'autres pour les peintres, de l'amour dans les tragédies, des héros modernes peu propres à la tragedie, des personnages allégoriques dans la peinture et dans la poésie, du vraisemblable, de la poésie du style, des Anciens, de leur langue, leur sculpture et enfin de la déclamation. Le second article rappelle rapidement les points importants du premier volume (donc du premier article) et examine en détail le contenu du second.

Il n'était pas nécessaire, vue la longueur du premier article, de rappeler les points du premier volume pourtant le journaliste le fait et il est intéressant de voir quels points sont retenus. Il rappelle le but de l'auteur, puis l'obligation aux peintres et poètes de choisir des sujets capables de toucher; enfin il termine sur le premier tome de Dubos en soulignant que les peintres, poètes et sculpteurs doivent avoir le respect des opinions vulgaires. Le journaliste explique par là que Dubos veut dire que le spectateur, doit être capable en regardant une peinture de faire immediatement des associations d'idées et il donne l'exemple du Nil pour illustrer sa pensée; voilà ce que dit le journaliste:

Notre ingénieux auteur loue le Bernin, d'avoir couvert d'un voile la tête de la statue qui représente le Nil, qu'il a d'ailleurs rendu reconnaissable par les attributs que les Anciens ont donne a ce fleuve.(177)

Le journal passe ensuite à l'examen du second Tome deux de Dubos sur lequel il s'etend avec détail sur la conception du "Génie" et n'hésite pas à citer longuement Dubos:

Le seul temps de la vie qui soit bien propre à faire acquerir leur perfection a l'oeil et a la main est le temps ou nos organes tant intérieurs qu'extérieurs achèvent de se former... C'est le temps qui s'écoule depuis l'âge de quinze ans jusques à l'âge de trente ans (178).

Le Génie de la poésie et celui de la peinture n'habite point dans un homme d'un temperament froid et d'une humeur indolente (179).

Le journal s'attarde sur les sections qui ont trait à l'influence du temps historique et du climat sur le "Génie"; comme nous le savons ces 
"réflexions" seront reprises par Montesquieu et beaucoup d'autres jusqu'au XIX ème siècle inclus. Enfin le journal consacre le reste de ses colonnes à examiner à l'appui de Dubos la réaction du spectateur devant une oeuvre d'art. Le journaliste reprend une citation déjà rendue célèbre douze ans plus tôt:

L'ouvrage plaît-il, ou ne plaitt-il pas? L'ouvrage est-il bon ou mauvais, en général? . . Raisonne-t-on pour savoir si un ragoat est bon ou s'il est mauvais? ...(183)

Et il est définitivement convaincu que la première réaction est la seule qui ait du poids lorsqu'il s'agit d'un jugement que l'on porte sur une oeuvre d'art. En 1732 on fait une distinction bien marquée entre les sciences et leurs progrès et les arts et leurs progrès. Les sciences et les arts étant d'un ordre différent, leurs progrès ne sauraient être jugés de la même manière.

En conclusion l'article est très élogieux. A la fin de son exposé le journaliste demande que le public l'excuse de ne pas avoir reconnu plus tôt les mérites de ce second tome. Le choix et le groupement des sections montrent la direction de l'intérêt que le journaliste détecte chez son public. La question du "jugement" par "sentiment" et la question du "Génie" semblent être vivement débattues et l'opinion du public va se stabiliser sans doute à la lumière d'un article comme celui--ci. Le développement de la presse périodique en France au XVIIIème siècle répond à un besoin, celui d'être au courant de tout et d'être en mesure de tout voir "en critique." Ce nouvel état d'esprit qui anime le public lettré de cette époque et qui consiste à faire un certain emploi critique de la raison en lui donnant un but pratique prend le nom "d'esprit philosophique." L'intelligence et la raison se mettent à l'unisson pour améliorer le bien-être individuelet collectif. L'esprit de libre examen né dans les années 1680, s'attarde dès le début du XVIIIème siècle sur les questions sociales aussi bien que métaphysiques. Les journaux périodiques de cette époque sont des auxiliaires importants d'étude et de propagande pour ce mouvement rationaliste. Le Journal des sçavans devient l'organe du gouvernement tandis que le Journal littéraire reste une entreprise privée. Les questions que les journalistes se posent sont les mêmes, à savoir: "Qu'y a-t-il de commun entre les ocuvres d'art pour qu'elles aient obtenu la faveur du public?" "Quel est le but de l'art?" 
"Quelle est sa raison d'être?." Ils en parlent en examinant les traités récemment publiés, dont Les Réflexions critiques sur la poésie et la peinture de l'Abbé Dubos. La peinture est bientôt préférée à la poésie du fait qu'elle plaît ou ne plaît pas au premier coup d'oeil, tandis que la poésie, elle, doit être lue pour être appréciée. Etant donné que la beauté d'une oeuvre d'art dépend du plaisir qu'elle procure au spectateur. et que celui-ci varie selon le spectateur, le relativisme gagne du terrain. Certains termes tels que "sens," "sensation," "sentiment," sont ré-examinés, redéfinis et traduisent une conception nouvelle, celle du "sixième sens," une conception essentiellement individualiste. Cette terminologie nouvelle suggère déjà un relativisme bien ancré qui se développera avec assurance transformant la société française d'une manière fondamentale.

John Carroll University

Hêlène Sanko

\section{BIBLIOGRAPHIE}

Dubos, Jean-Baptiste. Histoire des quatre Gordiens, 1695. Vindiciae pro quatuor Gordianorum historia, 1700. Les intérêts de l'Angleterre mal entendus dans la guerre présente, 1704 (il s'agit de la guerre de la Succession d'Espagne). Manifeste de Maximilien, électeur de Bavière contre Léopold, empereur d'Autriche, 1705. Histoire de la Ligue de Cambrai, 1709. Réflexions critiques sur la poésie et la peinture, 1719, 2 vol.; 1770, 3 vol. Histoire critique de l'établissement de la monarchie française dans les Gaules, 1734, 3 , vol. in $-4^{\circ}$. 
Feller, François Xavier de. Biographie universelle ou dictionnaire historique, nouvelle édition augmentée par M. Pégennes, Tome IV. Besançon: Outhenin Chalandre fils, 1842.

Grente, Georges. Dictionnaire des lettres françaises, le VXIII ème sièclc, Tome I. Paris: Fayard, 1960.

Le Journal littéraire, Tome XI, part I. La Haye: Johnson, 1720, [JL 20]; Tome IX, 1732, [JL 32].

Le Journal des sçavans (ou savans). Tome LXVI. Amsterdam: Jansson \& Rey, 1719. [JS 19], 1727 [JS 27].

Lombard, A. L'Abbé Dubos, un initiateur de la pensée moderne. Paris: Hachette, 1913.

Mustoxidi, Theodore M. Histoire de l'esthétique française (1700-1900). Paris: Champion, 1920. 